\section{Avaliação da Vigilância Epidemiológica em âmbito municipal}

\section{Evaluation of epidemiological surveillance in municipal ambit}

Eduardo Freese de Carvalho 1 Eduarda Ângela Pessoa Cesse 2 Maria Ilk Nunes de Albuquerque 3 Luciana Caroline Albuquerque 4 Luciana Santos Dubeux 5

1,2,4.5Centro de Pesquisas Aggeu Magalhães. Fundação Oswaldo Cruz. Departamento de Saúde Coletiva. Campus da Universidade Federal de Pernambuco. Av. Moraes Rego, s. n. Recife, PE, Brasil. CEP 50.670-420. freese@cpqam.fiocruz.br

1 Departamento de Medicina Social. Universidade Federal de Pernambuco. Recife, PE, Brasil.

3 Departamento de Enfermagem. Universidade Federal de Pernambuco. Recife, PE, Brasil.

\section{Resumo}

Objetivos: avaliar a vigilância epidemiológica (VE)

Objectives: to evaluate epidemiological surveillance (ES) and its specifics management at municipal level.

Methods: eleven municipalities were selected in the State of Pernambuco, Brazil, based on the geographical, demographic and management criteria. The level of implementation was estimated by way of interviews with key-players at three levels of ES: the headquarters, family health units (FHUs) and traditional health units (THUs), laying emphasis on features relating to infrastructure and the work process. Points were assigned for the selected variables, which, taken as a whole, allowed for classification of ES as: fully introduced, partially introduced, or not introduced. In order to evaluate management, the eleven municipal health secretaries were interviewed.

Results: ES was found to have been fully introduced in only $27 \%$ of municipalities and partially introduced in 45\%, having a better structure in larger-scale municipalities where there is full management of the municipal system. In these, of the three levels studied, it was found that the central level showed the greatest weaknesses, and that the FHUs performed better than the THUs. So far as management is concerned, although all the municipalities were certified to carry out ES activities, it was found that there are significant obstacles to its effective introduction.

Conclusions: ES still has shortcomings that hamper its full introduction at municipal level.

Key words Epidemiological surveillance, Services evaluation, Municipal management e as características específicas da gestão municipal.

Métodos: foram selecionados onze municípios de Pernambuco conforme critérios geográficos, porte populacional e condição de gestão. O grau de implantação foi estimado através de entrevistas com atores-chave em três níveis de atuação da VE: nível central, unidades de saúde da família (USF) e unidades de saúde tradicionais (UST), enfatizando aspectos relativos à infra-estrutura e ao processo de trabalho. Às variáveis selecionadas foram atribuídos pontos, cujo somatório, classificou a VE como: implantada, parcialmente implantada e não implantada. Para avaliar as características da gestão foram entrevistados os onze secretários municipais de saúde.

Resultados: a VE apresentou-se implantada em apenas $27 \%$ dos municípios e parcialmente implantada em $46 \%$, encontrando-se mais estruturada nos municípios de grande porte e com gestão plena do sistema municipal, onde, dos três níveis estudados, observou-se que o nível central apresentou mais fragilidades, e as USF, quando comparadas às UST, obtiveram melhor desempenho. Quanto às características de gestão, embora todos os municípios estivessem certificados para realizar ações de VE, foram identificados entraves significativos para sua efetiva realização.

Conclusões: A VE apresentou deficiências que comprometem a sua implantação plena no âmbito municipal.

Palavras-chave Vigilância epidemiológica, Avaliação dos Serviços, Administração municipal 


\section{Introdução}

A ampliação do conceito de Vigilância Epidemiológica (VE) está estabelecida na lei 8080/90 do Sistema Único de Saúde (SUS) como:

"... um conjunto de ações que proporcionam o conhecimento, a detecção ou prevenção de qualquer mudança nos fatores determinantes e condicionantes de saúde individual ou coletiva, com a finalidade de recomendar e adotar as medidas de prevenção e controle das doenças e dos agravos à saúde das populações" (Ministério da Saúde; 1990: 2). ${ }^{1}$

A partir dessa concepção ampliada, a VE passa a incorporar ações de observação, estudos epidemiológicos, avaliação e planejamento, relacionados ao combate das doenças transmissíveis e não-transmissíveis; além das atividades relacionadas às ações de controle, coleta de informações atualizadas, processamento, análise, interpretação, recomendações e disseminação das informações. 2

A orientação atual para o desenvolvimento da VE estabelece como prioridade o fortalecimento de sistemas municipais, dotados de autonomia técnicogerencial para enfocar problemas de saúde na sua área de abrangência. Tal reorientação tem contribuído para a mudança do modelo de atenção à saúde, antes voltado para ações clínico-assistenciais. Através de estratégias como o Piso Assistencial Básico (PAB), para financiamento das ações da Atenção Básica, e de programas como o Saúde da Família (PSF), o de Agentes Comunitários de Saúde (PACS), a proposta é que os municípios construam um novo modelo voltado para a consolidação da Vigilância à Saúde. ${ }^{3}$ Com isso, os propósitos, as estratégias e as ações de VE foram modificadas e reestruturadas nas três instâncias de gestão do sistema, a partir, principalmente, do estabelecimento de um processo de normatização capaz de uniformizar procedimentos e viabilizar a operacionalização das ações de VE.

Vale ressaltar que, apesar do arcabouço normativo-institucional, contido na Norma Operacional Básica (NOB-SUS 01/96), a implantação das ações de VE, especialmente no âmbito municipal, avançou lentamente, apresentando grandes dificuldades na operacionalização de suas práticas. ${ }^{4}$ Somente com as portarias 1.399 e 950, do Ministério da Saúde em 1999, é que a descentralização da VE foi efetivamente discutida e se definiram as atribuições das três instâncias de governo e a forma de financiamento das ações, através do Teto Financeiro de Epidemiologia e Controle de Doenças (TFECD).5-6

Com a proposta do novo modelo de atenção de integrar as ações de vigilância com os programas assistenciais, ${ }^{4}$ é necessário avaliar de que forma essa integração vem se reproduzindo nas realidades locais. Percebe-se uma nítida distância entre a operacionalização das ações de VE, na lógica de "apagar incêndios" e as ações assistenciais e individuais das unidades de saúde. 7

Neste sentido, algumas iniciativas têm surgido no intuito de fortalecer e qualificar a Vigilância em Saúde. Como exemplo, menciona-se o VIGISUS (Projeto de Estruturação do Sistema Nacional de Vigilância à Saúde), que visa tanto à melhoria da estrutura dos serviços, quanto à qualidade no desenvolvimento das ações de VE, transferindo não só responsabilidades para as três instâncias, como recursos financeiros e prestando assessoria técnica. 8 No que diz respeito à avaliação da VE, alguns instrumentos normativos têm contribuído para avaliação das ações de VE - os pactos firmados nas discussões tripartites, como os indicadores do Pacto de Indicadores da Atenção Básica ${ }^{9}$ e as metas da Programação Pactuada e Integrada da Vigilância à Saúde (PPIVS), 10 além de estudos acadêmicos.4-7,11,12

Entretanto, o estágio ainda incipiente em que se encontra a área de avaliação em saúde no Brasil13 depara-se com alguns obstáculos, tanto no que se refere à falta de cultura de avaliação por parte dos gestores na busca de conhecer os efeitos e os impactos produzidos pelos investimentos nas políticas e projetos, quanto pelo pouco conhecimento por parte dos profissionais sobre o método avaliativo. 14

Nesse contexto, para se tomar decisões no âmbito de uma política, projeto ou programa de saúde, de forma pactuada e democrática, é necessário que se realize constantemente um julgamento de valor da sua eficácia, eficiência e efetividade, considerando o contexto político, epidemiológico e organizacional em que estão inseridos. Isso visa à institucionalização da prática avaliativa nos serviços, como subsídio para o desenvolvimento de atividades de qualidade e compatíveis com a realidade local. 15

Fazendo uma reflexão sobre o fortalecimento dos sistemas municipais de saúde, proposto pelo SUS, em que pese a vigilância epidemiológica como um de seus instrumentos mais importantes de atuação, 16 e a carência de estudos avaliativos que contemplem a VE, objetivou-se nesse artigo, a partir de uma pesquisa realizada sobre o processo de municipalização da saúde, estimar o grau de implantação das ações de VE, além de identificar as características específicas da gestão da vigilância epidemiológica na esfera municipal. 


\section{Métodos}

Realizamos uma avaliação normativa, para identificar o grau de implantação e uma pesquisa qualitativa exploratória, para se conhecer as principais características de gestão da VE, baseando-se nos fundamentos de Contrandioupolos et al. 17 e Minayo. ${ }^{18}$

Foram selecionados 11 municípios conforme a mesorregião (área metropolitana do Recife, zona da mata, agreste e sertão), porte populacional - pequeno porte (municípios com menos de 50.000 habitantes), médio porte (população entre 50.000 e 150.000), grande porte (municípios com mais de 150.000 habitantes), e condição de gestão de acordo com a NOB/SUS-96.19 Além disso, foram incluídos na amostra alguns municípios pólo, segundo critério das "regiões de desenvolvimento" do governo do estado de Pernambuco. Sendo assim, o estudo englobou um município de cada porte por mesorregião, com exceção da zona da mata, que por não possuir município de grande porte ficou representada por dois municípios. Quanto à condição de gestão, foram totalizados seis municípios em gestão plena da atenção básica e cinco em gestão plena do sistema municipal de saúde. Assim, com esse conjunto de critérios, a amostra intencional totalizou 11 municípios: Camaragibe, Moreno, Paulista, Tamandaré, Palmares, Riacho das Almas, Caruaru, Gravatá, Petrolina, Salgueiro e Mirandiba.

Para verificar o grau de implantação da VE foram realizadas entrevistas estruturadas com atores de diferentes níveis de atuação da VE. No nível central (NC) foi entrevistado o coordenador do Serviço de Vigilância Epidemiológica e nos níveis locais realizou-se a entrevista com um médico ou enfermeiro das Unidades de Saúde da Família (USF) e Unidades de Saúde Tradicional (UST).

O instrumento utilizado foi proposto por Alves, 20 sendo adaptado para os objetivos do estudo. Foram eleitos ações e indicadores traçadores, baseados em definições da Fundação Nacional de Saúde (FUNASA), ${ }^{21}$ da PPI, 10 e do Pacto de Atenção Básica. ${ }^{9}$ Esse abrangeu variáveis de estrutura (recursos humanos, espaço físico, equipamentos e insumos) e de processo (notificação, busca ativa, investigação, diagnóstico, processamento e análise dos dados, disseminação das informações, controle, monitoramento, educação em saúde e pesquisa) dos três níveis de atuação da VE em âmbito municipal.

Atribuíram-se pontos específicos de acordo com a importância de cada variável e, a partir do somatório desses, definiu-se o grau de implantação. Assim, quando o município avaliado apresentava entre $80 \%$ e $100 \%$ de respostas positivas, a VE foi considerada implantada; quando o resultado atingia entre $70 \%$ e $79 \%$, foi enquadrada como parcialmente implantada; e quando apresentou uma proporção abaixo de $70 \%$, foi considerada não implantada.

Procedeu-se ainda a uma entrevista semi-estruturada com os Secretários Municipais de Saúde quando foram identificadas as características de gestão da vigilância epidemiológica, a partir das seguintes variáveis: certificação para desenvolvimento das ações de VE, desempenho dessas conforme as atribuições definidas na criação do serviço de vigilância epidemiológica, financiamento, vínculo profissional, além de possíveis encaminhamentos para os entraves apontados pelos entrevistados.

Para a análise dos dados considerou-se o porte populacional do município, a condição de gestão, além dos níveis de atuação, com o intuito de ponderar cada uma dessas características isoladamente.

Este estudo foi submetido ao Comitê de Ética do Centro de Pesquisas Aggeu Magalhães, sendo aprovado através do registro no 60/02. Além disso, os entrevistados assinaram o termo de consentimento livre e esclarecido ao aceitarem participar da pesquisa.

\section{Resultados}

Em relação ao grau de implantação, a avaliação realizada identificou que em apenas $27 \%$ do total dos municípios estudados a Vigilância Epidemiológica pode ser enquadrada na categoria implantada, em $46 \%$ a VE foi verificada como parcialmente implantada e nos $27 \%$ restantes como não implantada, no âmbito municipal (Figura 1).

Entretanto, ao avaliarmos separadamente o grau de implantação por porte populacional, condição de gestão e nível de atuação da VE, foi possível identificar relevantes informações em relação a tais características (Quadro 1).

\section{Figura 1}

Distribuição proporcional do grau de implantação em municípios de Pernambuco, em 2003.

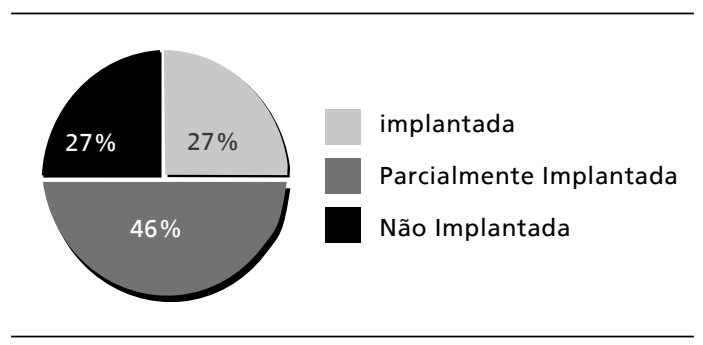


Quadro 1

Classificação do grau de implantação em municípios de Pernambuco, segundo mesorregião e porte populacional, 2003.

\begin{tabular}{|c|c|c|c|c|}
\hline \multirow[t]{2}{*}{ Mesorregiões } & Municípios & Porte populacional & Condição de gestão & Grau de Implantação \\
\hline & Camaragibe & Médio & Plena do Sistema Municipal de Saúde & \\
\hline \multirow[t]{2}{*}{ Metopolitana } & Moreno & Pequeno & Plena do Sistema Municipal de Saúde & \\
\hline & Paulista & Grande & Plena da Atenção Básica & \\
\hline \multirow{3}{*}{ Zona da Mata } & Tamandaré & Pequeno & Plena da Atenção Básica & \\
\hline & Palmares & Médio & Plena da Atenção Básica & \\
\hline & Rio das Almas & Pequeno & Plena da Atenção Básica & \\
\hline \multirow[t]{3}{*}{ Agreste } & Caruaru & Grande & Plena do Sistema Municipal de Saúde & \\
\hline & Gravatá & Médio & Plena do Sistema Municipal de Saúde & \\
\hline & Petrolina & Grande & Plena do Sistema Municipal de Saúde & \\
\hline \multirow[t]{2}{*}{ Sertão } & Salgueiro & Médio & Plena da Atenção Básica & \\
\hline & Mirandiba & Pequeno & Plena da Atenção Básica & \\
\hline
\end{tabular}

Implantada

Parcialmente Implantada

Não Implantada

Nos municípios de pequeno porte a VE não foi classificada como implantada em nenhum dos municípios estudados. Nos demais a VE pode ser considerada implantada em 50\% dos municípios de médio porte e em $66,7 \%$ dos municípios de grande porte (Figura 2).

Do mesmo modo, ao compararmos os municípios de acordo com sua condição de gestão, identifi-

\section{Figura 2}

Distribuição proporcional do grau de implantação dos municípios de Pernambuco, segundo porte do município, em 2003.

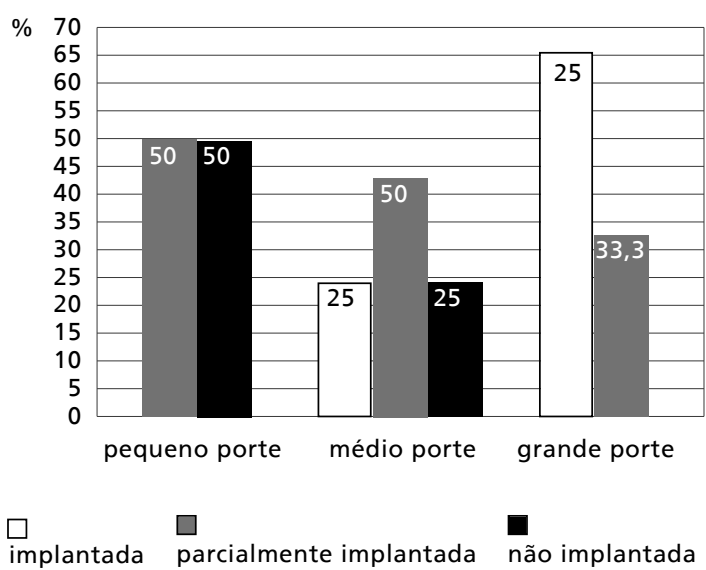

camos que a maioria dos municípios $(66,7 \%)$ em gestão plena da atenção básica apresentou a VE como parcialmente implantada, não se verificando nenhum município com a ação implantada. Por outro lado aqueles em gestão plena do sistema municipal apresentaram a VE predominantemente $(60 \%)$ implantada (Figura 3).

Figura 3

Grau de implantação da Vigilância Epidemiológica em municípios de Pernambuco, segundo condição de gestão, em 2003.

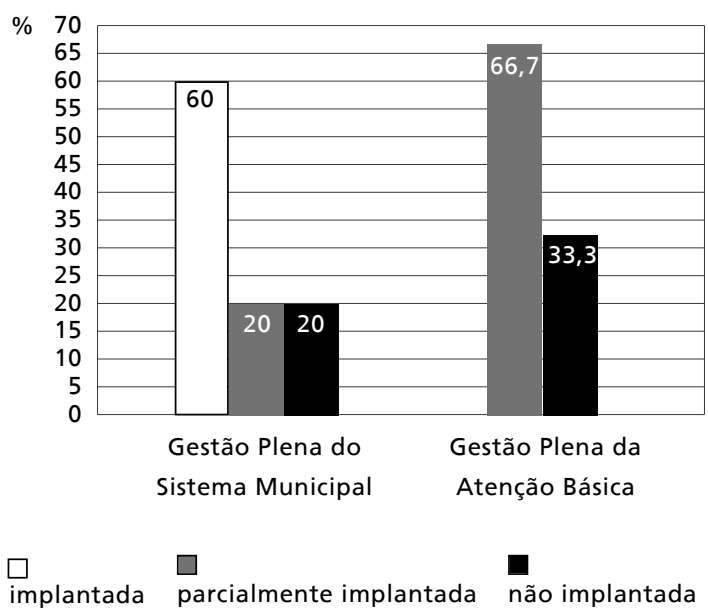




\section{Figura 4}

Grau de implantação da Vigilância Epidemiológica por nível de atuação da Vigilância Epidemiológica em municípios de Pernambuco, em 2003.

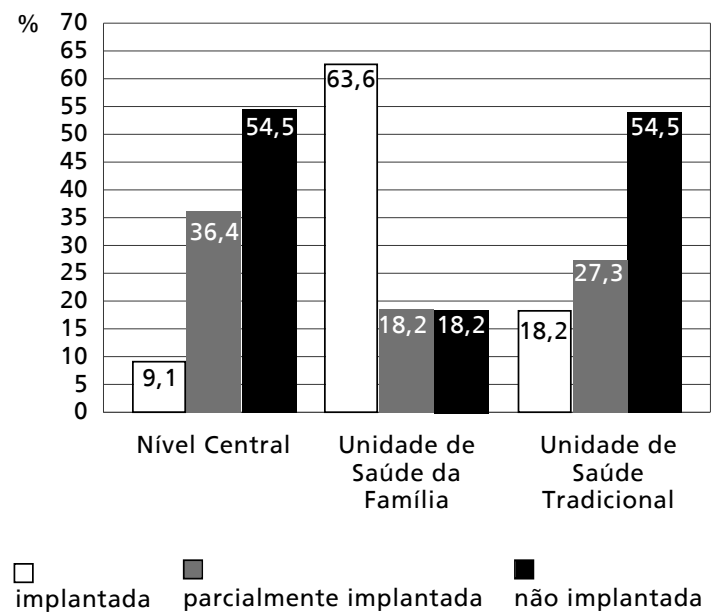

Quanto a avaliação do grau de implantação da VE por nível de atuação (nível central, unidade de saúde da família e unidade de saúde tradicional) é possível identificar características específicas de cada um desses, quanto às variáveis de estrutura e de processo de trabalho da VE.

Assim, analisando apenas o desempenho do nível central observou-se, que apenas em 9,1\% dos municípios a VE pode ser classificada como implantada, e em $36,4 \%$ parcialmente implantada. $\mathrm{Na}$ maioria dos municípios $(54,4 \%)$ a VE está classificada na condição não implantada (Figura 4).

Entre as atividades necessárias para a execução da VE, destaca-se que apesar do universo dos municípios confirmar as principais atividades de investigação (tais como investigação oportuna de sarampo, de casos de doenças de notificação compulsória, de óbito materno e infantil) e de monitoramento (de doenças diarréicas agudas, mortalidade materna e infantil), apenas dois municípios responderam que analisam os dados, através da construção de mapas de risco e gráficos de tendências. Além disso, nenhum município indicou retroalimentar os laboratórios em relações às informações dos casos. Quanto à estrutura, observou-se que em vários municípios, embora grande parte dos gestores seja especialista em saúde pública, há ausência de técnicos de nível superior; bem como de normatização interna escrita e equipamentos de informática (computadores e softwares como Tabwin e Epi-info).

Já ao considerar os níveis locais, o estudo identi- ficou que enquanto na maioria das unidades de saúde da família $(63,6 \%)$ a VE se apresentou como implantada, as unidades de saúde tradicional atingiram predominantemente a classificação não implantada $(54,5 \%)$, estando implantada em apenas $18,2 \%$ dessas (Figura 4).

Quanto às atividades relativas à VE, as unidades de saúde da família indicaram realizar a maioria delas, embora algumas atividades importantes como a construção de indicadores para análise dos dados tenha sido pouco relatada. Diferentemente, as unidades de saúde tradicionais desempenham menor número de atividades, sendo omitidas, por grande parte dos profissionais, algumas atividades essenciais para a efetiva realização da VE, tais como: a consolidação e análise dos dados, a notificação de agravos e a disseminação das informações. Porém, apesar da considerável discrepância no desempenho dos níveis locais de atuação da VE, as deficiências na infra-estrutura (ausência de computadores e softwares) são comuns tanto nas unidades de saúde da família quanto nas unidades de saúde tradicionais na maioria dos municípios.

Em seguida, foram identificadas algumas características de gestão da vigilância epidemiológica, que embora não sejam suficientes para determinar o grau de implantação da VE, contribuem para demonstrar o contexto em que as ações de vigilância epidemiológica são realizadas.

Dessa forma, ao considerarmos a certificação para o desenvolvimento das ações de epidemiologia e controle de doenças, apenas um entrevistado não soube responder se o município está certificado para desenvolver tais ações. Entretanto, o mesmo representa um município de grande porte, habilitado em gestão plena do sistema municipal, com a VE classificada como implantada e que se destacou por realizar a maioria das ações de vigilância epidemiológica. Quanto aos demais, $40 \%$ identificou o ano de início da certificação entre 1996 e 2001.

Independente do grau de implantação alcançado, a maioria dos entrevistados apontou que as atividades de VE desenvolvidas em seu município estão de acordo com as atribuições definidas na criação do serviço, com exceção de um secretário de saúde, cujo município apresentou a VE não implantada. Esse indicou não haver coerência entre estado e união no repasse das informações para o município, que é o executor das ações.

Quanto ao valor correspondente ao Teto Financeiro para Epidemiologia e Controle de Doenças, (TFECD) todos os secretários de saúde afirmaram conhecê-lo, com exceção de um que embora tenha afirmado não conhecer, apontou que é insuficiente 
para a efetiva realização das ações de VE. Justificouse que o teto disponível obriga o município a disponibilizar uma contra-partida superior ao preconizado, dificultando a contratação de recursos humanos; e que foram incorporadas outras endemias (como a leishmaniose e esquistossomose) à rotina de atuação da VE, sem incremento dos recursos financeiros.

Nesse aspecto, apenas um secretário, representante de um município de pequeno porte, em gestão plena do sistema municipal de saúde e que teve a VE enquadrada como não implantada, indicou que o teto é suficiente.

Conseqüentemente, ao serem questionados em relação ao desempenho da VE, 55\% dos entrevistados o consideram como "bom", embora $27 \%$ tenham indicado desempenho "regular" (Figura 5). Todos os secretários de saúde, inclusive aqueles que classificaram as ações de VE como "ótima" (18\%), justificaram a sua percepção considerando o desempenho das ações e atividades desenvolvidas.

Nesse sentido, algumas afirmativas se destacaram como um paradoxo ao retratar a percepção dos entrevistados. Ao indicar que a VE tem "bom" desempenho, um secretário de saúde responsável por um município de pequeno porte, gestão plena do sistema municipal e com a VE classificada como não implantada respondeu:

"... a vigilância atende às demandas

regulamentares, porém para ser ótima

precisava dar um passo além... não apenas

apagar incêndios".

Outros gestores apontaram para a necessidade de captação de profissionais especializados que cumpram a carga horária de trabalho, como também de mais equipamentos e veículos. Além disso, alguns entrevistados ponderaram sobre a qualidade das ações de VE, como afirmou o secretário de saúde de

\section{Figura 5}

Opinião dos gestores quanto ao desempenho da Vigilância Epidemiológica em municípios de Pernambuco, em 2003.

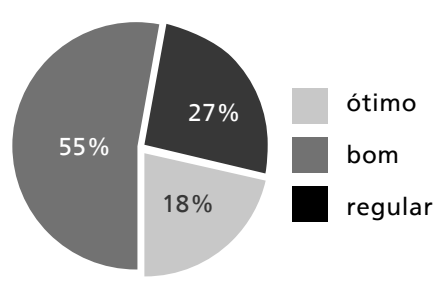

um município de pequeno porte, gestão plena da atenção básica e com a VE não implantada:

"... o pessoal disponível ainda não trabalha como deveria na notificação... falta interesse e nas orientações eles não dão importância".

Outro profissional aponta também:

"As metas da pactuação vêm sendo cumpridas, mas ainda sem qualidade $e$ efetividade que se deseja".

De outro modo, um entrevistado ao considerar que a Vigilância Epidemiológica de seu município apresenta "ótimo" desempenho declarou:

"O município é o único no estado que realiza notificação por ocorrência, além de investigar $100 \%$ dos casos de óbitos por causa desconhecida... a equipe é excelente". (Secretário de Saúde de um município de grande porte, gestão plena do sistema municipal e com a VE implantada).

Entretanto, 72,7\% dos entrevistados apontaram a existência de entraves na execução das ações de VE, sendo os mesmos de natureza apenas financeira para maioria $(50,0 \%)$ dos secretários de saúde; decorrentes de problemas administrativos e financeiros como informou $37,5 \%$ dos entrevistados ou do conjunto de problemas administrativos, políticos e financeiros que foi apontado por $12,5 \%$ desses gestores.

Sendo assim, todos os secretários opinaram sobre a necessidade de melhoria do desempenho da VE em seu município, indicando possíveis soluções para os entraves e apontando algumas mudanças introduzidas na atual gestão.

Nesse sentido, os encaminhamentos permearam principalmente sobre a contratação e capacitação de recursos humanos especializados e com carga horária suficiente para desempenho das ações; a melhoria da articulação entre as gerências regionais de saúde e os municípios; o aumento das discussões com a Comissão Intergestores Bipartide (CIB) e com o Conselho dos Secretários Municipais de Saúde (COSEMS); a revisão do TFECD; além de questões operacionais como aquisição de veículos exclusivos para o setor, instalações físicas e equipamentos de informática.

Nesse contexto, os gestores foram questionados em relação às possíveis mudanças introduzidas pela a atual gestão na estrutura e processo de trabalho da VE. As iniciativas relatadas corresponderam às ações relativas ao processo de investigação (investigação de $100 \%$ dos óbitos com causa não definida); à disseminação das informações (retroalimentação das unidades de saúde com dados epidemiológicos) 
e ao monitoramento (maior controle dos indicadores e idealização de uma sala de situação). Além disso, foram mencionadas ações como a desvinculação do Programa Nacional de Imunização à VE, bem como a ampliação das atividades de vigilância epidemiológica para doenças crônicas não-transmissíveis, através do acompanhamento e diagnóstico dos fatores de risco para câncer e tabagismo; e da organização de um projeto direcionado para diabetes, hipertensão e agravos prioritários, como hanseníase e tuberculose.

Quanto ao incremento da infra-estrutura, os entrevistados citaram a implementação de sistemas de informação, SINAN, SINASC e SIM, a aquisição de equipamentos e veículos, a contratação de técnicos para VE e equipes de informática; além da criação de um centro de vigilância à saúde com maior sensibilização dos profissionais da rede de saúde pelos técnicos de vigilância epidemiológica do nível central em âmbito municipal.

\section{Discussão}

Apesar de todo o aparato legal e oficial, que subsidia as ações de Vigilância Epidemiológica (VE), o que ainda se observa é um descompasso entre o discurso e a efetiva utilização da VE, enquanto parte integrante de um sistema e co-responsável na definição de ações prioritárias das políticas de saúde.

Esse fato foi constatado quando ações elementares de vigilância encontraram-se fragilizadas na maioria dos municípios pesquisados, embora se reconheçam limitações metodológicas no estudo. Tais limitações se referem à opção pela avaliação normativa, que se propõe a confrontar as normas com o que de fato é realizado, sem, no entanto, analisar o contexto em que as ações são desenvolvidas e/ou o impacto delas.

Ao analisarmos os resultados obtidos, a partir dos critérios definidos para a escolha dos 11 municípios, observamos que o porte populacional e a condição de gestão foram determinantes no grau de implantação da VE, uma vez que os municípios de grande porte e em Gestão Plena do Sistema de Saúde obtiveram melhor desempenho. Tal fato reitera a NOB-SUS/96, quando diz que a certificação do município nessa condição de gestão estabelece como pré-requisito a comprovação da estruturação de serviços e atividades de vigilância epidemiológica e de controle de zoonoses. ${ }^{19}$ Todavia, o terceiro critério utilizado, que é a característica geográfica, não influenciou no resultado, ficando cada mesorregião com seus municípios distintamente classificados.
Tomando-se como referência a portaria GM/1.172/04,22 que normatiza como funções da VE no âmbito municipal: a notificação dos agravos; a investigação, a busca ativa, o provimento da realização de exames laboratoriais, o acompanhamento e a avaliação dos procedimentos laboratoriais, a coordenação e a execução das ações de vacinação, o monitoramento da mortalidade infantil e materna, a gestão e/ou gerência dos sistemas de informação epidemiológica, a divulgação de informações, as análises epidemiológicas e a capacitação de recursos humanos, nos municípios certificados de fato, tais ações foram descentralizadas, não sendo mais de responsabilidade estadual. Porém, algumas delas, como o processamento e análise dos dados e a disseminação das informações ainda não fazem parte da rotina dos serviços, comprometendo a efetividade da "informação para ação", um dos objetivos da VE. 23

Goldbaum, 24 relata que esse acontecimento se justifica pela carência de profissionais, pela falta de capacidade técnica e pelo fato dos serviços não possuírem uma infra-estrutura razoável para o desempenho da VE. De acordo com Villa,25 a descentralização da VE para as unidades básicas de saúde é plausível, desde que se ofereçam profissionais qualificados e infra-estrutura compatível com suas atribuições. Certamente, falhas estruturais podem inviabilizar o desenvolvimento das ações, todavia, focando os resultados deste estudo no grau de implantação dos componentes estrutura e processo, observou-se que a infra-estrutura dos serviços está implantada na metade dos municípios, enquanto que nas questões referentes ao processo, a VE esteve implantada em apenas um deles. Ou seja, a infra-estrutura necessária para um bom desempenho da VE não se constitui um problema para realização de suas ações, uma vez que um percentual de $50 \%$ dos municípios estudados conta com a estrutura mínima para tanto. O que se percebe, em última instância, é uma clara dificuldade de se conseguir romper com a hegemonia das intervenções assistenciais, extremamente necessárias, porém totalmente desintegradas das práticas de vigilância. ${ }^{7}$

Outro fato bastante pertinente foi a falta de definição, seja nos regimentos internos, seja na própria rotina dos serviços, quanto às atribuições que competem a cada um dos níveis de atenção (nível central, unidades de saúde da família e unidades de saúde tradicionais). Houve uma certa dificuldade no julgamento de "quem deveria fazer o quê". Numa visão global, verificou-se que algumas das ações que deveriam estar descentralizadas para as unidades de saúde, - uma vez que têm o foco no território, como 
a notificação, a busca ativa dos casos suspeitos e dos faltosos à vacinação anti-rábica - a investigação das mortes maternas e infantis e o monitoramento de alguns agravos, ainda fazem parte da rotina do nível central na maioria dos municípios, principalmente nos de pequeno porte. Um aspecto que pode estar relacionado a isso, é o fato da sobrecarga relatada por alguns dos profissionais entrevistados nas unidades básicas, no que se refere à quantidade de planilhas que têm que preencher diariamente e à própria rotina de atendimentos, o que dificulta a introdução das atividades de vigilância.

Passos $^{7}$ afirma que se faz necessário desmistificarem as ações de VE, nas unidades básicas, vistas enquanto preenchimento de papéis para enviar ao nível central, sem sequer saberem da importância dessas informações para as suas ações. Para isso, devem ser elaborados instrumentos que contenham dados imprescindíveis e fáceis de preencher. Além disso, é necessário que as unidades valorizarem as informações que repassam para o nível central, sendo fundamental também um retorno da análise de parte desses dados. Dessa forma, apontamos para a necessidade dos níveis locais realizarem a análise de uma parcela importante dos dados obtidos nessa instância, na lógica da descentralização do planejamento da gestão e da avaliação da saúde.

Nesse contexto, o melhor desempenho das Equipes de Saúde da Família (ESF), quando comparadas às Unidades de Saúde Tradicionais, talvez tenha sido porque o modelo proposto para o PSF com práticas voltadas para ações de promoção à saúde e prevenção, além da responsabilidade sanitária, permite uma maior aproximação com as atividades da VE. Por outro lado, a própria característica das unidades tradicionais, com ênfase nas especialidades, na demanda espontânea e nas atividades curativas não seja condizente com o novo modelo de atenção à saúde e com as diretrizes da atenção primária.

Ao confrontarmos os dados desta pesquisa com um estudo realizado em 948 unidades de saúde situadas em 98 municípios do Brasil, quando se avaliou o processo de trabalho da VE no que diz respeito ao fluxo de informações, análise dos dados e realização de investigação epidemiológica, percebe-se a convergência dos resultados. Constatou-se que 53,7\% das unidades não seguiam as normas mais elementares das atividades de VE e, quanto à capacitação de recursos humanos, de 3000 profissionais que foram treinados em todos os Estados, os mesmos foram encontrados em apenas um terço das unidades. 11

Nesse mesmo estudo11 observa-se que, embora as ações de vigilância epidemiológica estejam historicamente voltadas para as doenças transmissíveis e os indicadores de mortalidade em relação a essas doenças apresentarem uma importante redução, ainda assim, o modelo de vigilância proposto apresenta-se fragilmente consolidado. Além disso, para a admissão de ações de monitoramento e vigilância das doenças crônicas e de seus fatores de risco, faz-se necessária a produção da informação epidemiológica e sua utilização pelos níveis locais, para a partir daí saírem as estratégias de intervenção.

Outro estudo realizado no Estado de Pernambuco identificou que a mortalidade por doenças crônicas não transmissíveis é crescente, sendo ainda a terceira causa de internação no estado. ${ }^{26}$ Tal contexto reforça a importância da adequação da vigilância epidemiológica no sentido de apoiar o desenvolvimento de ações voltadas para esse conjunto de agravos. Cabe destacar as iniciativas apontadas pelos secretários de saúde dos municípios avaliados nesse estudo, no sentido de contribuir para o desenvolvimento de ações compatíveis com o padrão epidemiológico atual.

Ainda que as competências municipais tenham assumido as ações executivas da VE, dificuldades estruturais do sistema de saúde e da própria VE nos serviços têm demonstrado um caráter meramente descritivo, restrito à coleta de dados, e por vezes, à transmissão desses dados a outros níveis. 27 Os dados coletados, geralmente, são das doenças de notificação compulsória e raramente são analisados e utilizados para tomada de decisões, particularmente na instância municipal.

Portanto, é importante também haver uma desconstrução das práticas clínico-individualistas, enraizadas na maioria dos profissionais e, provavelmente, só a partir desse processo será possível realizar as mudanças necessárias à incorporação do novo modelo e a criação de uma nova consciência sanitária.

\section{Agradecimentos}

Agradecemos à Fundação de Amparo à Ciência e Tecnologia de Pernambuco (FACEPE) o financiamento do desenvolvimento do Projeto "Análise do perfil das doenças crônicas não-transmissíveis em mesorregiões e municípios de Pernambuco: subsídios para uma proposta de vigilância epidemiológica", através do Programa de Ciência e Tecnologia em Saúde - C\&T Saúde, Edital 02.2002. 


\section{Referências}

1. Ministério da Saúde. Lei n ${ }^{\circ} 8.080$, de 19 de setembro de 1990. Dispõe sobre as condições para a promoção, proteção e recuperação da saúde, a organização e o funcionamento dos serviços correspondentes e dá outras providências. Diário Oficial República Federativa do Brasil [DOU] 1990 set 20; Seção 1: 18055-9.

2. Waldman EA. Vigilância epidemiológica como prática de saúde pública [tese doutorado]. São Paulo: Faculdade de Saúde Pública da Universidade de São Paulo; 1991.

3. Teixeira CF, Paim JS, Vilasboas AL. SUS, modelos assistenciais e vigilância da saúde. Inf Epidemiol SUS 1998; 7: 8-28.

4. Silva Júnior JB. Epidemiologia em serviço: uma avaliação de desempenho do Sistema Nacional de Vigilância em Saúde [tese doutorado]. Campinas: Faculdade de Ciências Médicas da Universidade Estadual de Campinas; 2004.

5. Ministério da Saúde. Portaria GM n 1.399 , de 15 de dezembro de 1999. Regulamenta a NOB SUS 01/96 no que se refere às competências da União, estados, municípios e Distrito Federal, na área de epidemiologia e controle de doenças, define a sistemática de financiamento e dá outras providências [on line] 1999. Disponível em URL: Http://portal.saude.gov.br/ portal/svs/visualizar_texto.cfm?idtxt=21247. [2002 out 17].

6. Ministério da Saúde. Portaria $n^{\circ} 950$, de 23 de dezembro de 1999. Define os valores anuais per capita e por quilômetro quadrado, relativos aos recursos federais destinados à composição do Teto Financeiro de Epidemiologia e Controle de Doenças TFECD, de cada um dos estratos previstos no art. 14 da Portaria GM/MS n. ${ }^{\circ} 1.399 / 99$. Disponível em URL: Http://portal.saude.gov.br/portal/svs/visualizar_texto.cfm? idtxt=21245 [2005 Nov 30].

7. Passos LMR. Assistir e vigiar: as ações de vigilância epidemiológica na unidade básica de saúde: situação atual e perspectivas [tese doutorado]. Ribeirão Preto: Escola de Enfermagem de Ribeirão Preto da Universidade de São Paulo; 2003.

8. Ministério da Saúde. Secretaria de Vigilância à Saúde. Projeto VIGISUS II. Brasília (DF); 2004.

9. Ministério da Saúde. Secretaria de Políticas de Saúde. Departamento de Atenção Básica. Secretaria Executiva. Departamento de Informática do SUS. Pacto de Indicadores de Atenção Básica - 2002. [on line]. Disponível em URL: Http://www.dtr2003. saude.gov.br/pacto2002.htm [2002 Out 11].

10. FUNASA (Fundação Nacional de Saúde). Programação Pactuada Integrada - PPI/ECD 2002 - Região Nordeste parte 2, 2002. [on line]. Disponível em URL: Http://www.funasa.gov.br /epi/epi/epi/ppi00.htm [2002 out 17].

11. Carvalho MS, Marzocchi KBF. Avaliação da prática de vigilância epidemiológica nos serviços públicos de saúde no Brasil. Rev Saúde Pública 1992; 26: 34-57.
12. Mendes MFM, Freese de Carvalho E, Guimarães, MJB. Núcleos de epidemiologia em hospitais de alta complexidade da rede pública de saúde situados no Recife, Pernambuco: avaliação da implantação. Rev Bras Saude Matern Infant 2004; 4: 435-47 [on line]. Disponível em: $<$ http://www.scielo.br/scielo.php?script=sci_arttext\&pid=S $1519-38292004000400013 \& \operatorname{lng}=$ pt\&nrm $=$ iso $>[2005$ nov 30].

13. Matida AH, Camacho LAB. Pesquisa avaliativa e epidemiologia: movimentos e síntese no processo de avaliação de programas de saúde. Cad Saúde Pública 2004; 20: 37-47.

14. Novaes HMD. Epidemiologia e avaliação em serviços de atenção médica: novas tendências na pesquisa. Cad Saúde Pública 1996; 12 [Suppl 2]: 7-12.

15. Freese de Carvalho E, Sampaio J, Cesse E. Avaliação do Sistema Único de Saúde. In: Augusto LGS, Carneiro R, Martins PH. Abordagem ecossistêmica em saúde. Recife: Ed Universitária da UFPE; 2005.

16. FUNASA (Fundação Nacional de Saúde). Guia de vigilância epidemiológica. Brasília (DF); 2002. v. 1.

17. Contandriopoulos A-P, Champagne F, Denis J-L, Pineault R.. A avaliação na área da saúde: conceitos e métodos. In: Hartz, ZMA. Avaliação em saúde: dos modelos conceituais à prática na análise da implantação de programas. Rio de Janeiro: FIOCRUZ; 1997.

18. Minayo MCS. O desafio do conhecimento: pesquisa qualitativa em saúde. 7. ed. São Paulo: Hucitec; 2000.

19. Ministério da Saúde. Gestão municipal de saúde: leis, normas e portarias atuais. Rio de Janeiro; 2001.

20. Alves CKA. Proposta de avaliação do grau de implantação das ações de vigilância epidemiológica em âmbito municipal [monografia especialização]. Recife: Centro de Pesquisas Aggeu Magalhães da Fundação Oswaldo Cruz; 2003.

21. FUNASA (Fundação Nacional de Saúde). Avaliação dos indicadores de qualidade das atividades de vigilância epidemiológica e ambiental, 2000 [on line]. Disponível em URL: Http://www.funasa.gov.br/epi/epi_avalia.htm [2002 out 17].

22. Ministério da Saúde. Portaria GM 1.172/04, de 15 de junho de 2004. Regulamenta a NOB SUS 01/96 no que serefere às competências da União, Estados,Municípios e Distrito Federal, na área de Vigilância em Saúde, define a sistemática de financiamento e dá outras providências. Diário Oficial República Federativa do Brasil [DOU] 2004 jun 17; Seção 1: 58-9.

23. Cesse EAP, Freese de Carvalho E. Vigilância epidemiológica: evolução do conceito e institucionalização de práticas no contexto municipal. In: Freese de Carvalho E, organizador. Municípios: a gestão da mudança em saúde. Recife: Ed Universitária da UFPE; 2004. p. 91-110.

24. Goldbaum M. Epidemiologia e serviços de saúde. Cad Saúde Pública; 199612 [Supl 2]: 95-8.

25. Villa TCS. A vigilância epidemiológica e a perspectiva de trabalho no território - Secretaria Municipal de Saúde Ribeirão Preto. Rev Latinoam Enf 2002; 10: 21-7. 
26. Freese de Carvalho E, coordenador. Análise do perfil das doenças crônicas não-transmissíveis em mesorregiões e municípios de Pernambuco: subsídios para uma proposta de vigilância epidemiológica: relatório final. Recife: Centro de Pesquisas Aggeu Magalhães; 2004.
27. Lessa I. Doenças crônicas não transmissíveis: bases epidemiológicas. In: Rouquayrol MZ. Epidemiologia e saúde. Rio de Janeiro: MEDSI; 1999. p. 285-99.

Recebido em 16 de agosto de 2005

Versão final apresentada em 1 de dezembro de 2005

Aprovado em 9 de dezembro de 2005 